\title{
La rupture climatique de 1987 en France : quels effets sur l'humidité des sols?
}

\author{
Lisa Sick, Albin Ullmann*, Pascal Roucou \\ BIOGEOSCIENCES (UMR6282), équipe CRC, Université de Bourgogne, Dijon, France
}

\begin{abstract}
Résumé - Cet article présente les caractéristiques spatio-temporelles de l'humidité des sols à l'échelle de la France entre deux sous-périodes (1959-1987 et 1988-2019). Les principaux résultats montrent un assèchement généralisé des sols, surtout au printemps et en été, notamment pour trois secteurs : le sud-ouest, le nord-est et le pourtour méditerranéen. L'assèchement des sols peut s'expliquer par un important réchauffement des températures, généralisé et significatif à l'échelle de l'ensemble du territoire, mais bien plus marqué dans le sudouest et le nord-est, au printemps et en été. Cette élévation importante des températures entre les deux souspériodes semble expliquer une très forte hausse de la demande évaporatoire de l'atmosphère, extrêmement marquée du printemps à la fin de l'été. Cette augmentation de l'évapotranspiration potentielle est ici présentée comme un des facteurs déterminant de l'assèchement des sols et de l'aggravation du risque de sécheresses agricoles liés au réchauffement climatique.
\end{abstract}

Mots-clés : sécheresse du sol, hausse des températures, demande évaporatoire de l'atmosphère, France.

\begin{abstract}
Climatic shift in France in 1987: which impacts on soil moisture? This paper presents the spatiotemporal characteristics of soil moisture across France between two sub-periods (1959-1987 and 1988-2019). The main results show a general drying out of soils, especially in spring and summer and in particular for three sectors: the south-west, the north-east and the Mediterranean area. The drying out of soils can be explained by a significant warming of temperatures, generalized and significant at the whole French scale but is much stronger in the southwest and the northeast and in spring and in summer. This significant warming of temperatures between the two sub-periods seems to explain a very strong increase in the evaporative demand of the atmosphere, extremely marked from spring to the end of summer. This increase in potential evapotranspiration is presented here as one of the determining factors in the drying out of soils and in the aggravation of the risk of agricultural droughts linked to global warming.
\end{abstract}

Keywords: soil drought, temperature increase, potential evapotranspiration of the atmosphere, France.

\footnotetext{
* $\overline{\text { Auteur de correspondance }}$ : albin.ullmann@u-bourgogne.fr
}

\section{Introduction}

Depuis de nombreuses décennies, les rapports du GIEC alertent sur les conséquences de la hausse de la concentration des gaz à effet de serre qui modifient les caractéristiques naturelles du climat (IPCC, 2013, 2021). Ces modifications dans la composition de l'atmosphère entraînent notamment un accroissement des risques climatiques et des événements extrêmes qui pourraient être de plus en plus fréquents au $21^{\mathrm{e}}$ siècle (Forzieri et al., 2016). La hausse des températures est le changement le plus directement lié à l'augmentation des émissions et de la concentration en gaz à effet de serre. Ce réchauffement n'est pas linéaire. En France, les travaux de Brulebois et al. (2015) montrent une brusque élévation des températures en 1987 (une « rupture climatique ») qui s'est traduit par des valeurs minimales et maximales moyennes se réchauffant respectivement de $0,9^{\circ} \mathrm{C}$ et $1,1^{\circ} \mathrm{C}$ entre la période 1959-1986 et la période 1987-2009. Les tests non paramétriques de Mann-Kendall, Pettitt et SMWDA (Split moving-window distance analysis) ont montré que les ruptures de stationnarité dans les 
températures maximales et minimales sont significatives pour la quasi-totalité des stations de mesure utilisées couvrant l'ensemble du territoire français (Brulebois et al., 2015). Plusieurs hypothèses sont avancées pour expliquer l'existence de ce saut des températures : le changement de la phase négative à positive de l'oscillation nord atlantique (NAO) fin des années 1980 (Cassou, 2004); l'augmentation de la concentration des GES et donc du forçage radiatif ou encore la réduction des émissions d'aérosols d'origine anthropique lors des années 1980 qui favoriserait l'augmentation des températures en raison de l'abaissement de leur effet refroidissant (Brulebois et al., 2015 ; Seo et al., 2020). Finalement, l'origine du saut des températures de 1987 est probablement multifactorielle et semble liée à la variabilité interne du climat, combinée à des changements de concentration atmosphérique en GES et en aérosols.

Un des principaux aléas climatiques associé aux risques sur les agrosystèmes est la sécheresse, surtout lorsque celle-ci affecte les sols. Cet aléa pédologique est d'autant plus important qu'il est sensible au changement climatique, notamment au travers du réchauffement des températures et des changements dans les précipitations. Au cours du $21^{\mathrm{e}}$ siècle, Vidal et al. (2012) estiment par exemple que toutes les caractéristiques spatiotemporelles des épisodes de sécheresse devraient augmenter considérablement, avec des changements plus marqués pour les sécheresses agricoles. Ces événements particuliers et complexes ont par le passé entraîné d'importants retards dans les calendriers des semis mais surtout des déficits drastiques des rendements de céréales et des prairies (Van der Velde et al., 2012 ; Raymond et al., 2018 ; Rapport AGRESTE, 2018, 2019, 2020). Si de nombreuses études d'impact du changement climatique sur le cycle de l'eau ont été menées, celles-ci concernaient surtout les précipitations et les débits des rivières alors que celles consacrées à l'humidité des sols superficiels, dans lesquels les plantes puisent l'eau, sont plus rares. Le Projet Climsec lancé en 2008 par la Direction de la Climatologie de Météo France a permis de caractériser l'impact du changement climatique sur la ressource en eau, et plus particulièrement sur l'humidité des sols. Une typologie des sécheresses en métropole sur la période 1959-2008 a ainsi été créée, de même qu'un diagnostic sur leurs évolutions au cours du $21^{\mathrm{e}}$ siècle (Soubeyroux et al., 2011).

Le principal objectif de cette étude est de décrire les effets de la brusque élévation des températures observée en 1987 sur la variabilité spatiotemporelle de l'humidité des sols à l'échelle de l'ensemble du territoire français. Pour cela, les analyses se focaliseront notamment sur les liens entre les températures et la demande évaporatoire de 1'atmosphère (ETP).

\section{Données et méthodes}

Les données sont issues de la chaîne de modélisation SAFRAN-ISBA-MODCOU (SIM2). La chaîne est composée de trois modules de forçage atmosphérique (SAFRAN), d'un schéma de surface (ISBA) permettant de simuler les transferts d'eau et de chaleur dans le sol, la végétation, la neige, et l'hydrologie superficielle (drainage et ruissellement), ainsi qu'un module hydrologique (MODCOU) (Habets et al. 2008 ; Soubeyroux et al., 2008). En utilisant des observations de surface et des analyses issues de modèles atmosphériques, SAFRAN calcule en sortie des variables météorologiques alimentant le schéma de surface ISBA qui produit des bilans de surface, en particulier le ruissellement et le drainage profond. Les transferts hydrologiques peuvent ensuite être simulés par MODCOU. La chaîne SIM est utilisée en mode temps réel pour produire une analyse des variables météorologiques de surface à partir de plus de 1000 stations météorologiques pour la température et l'humidité de l'air à $2 \mathrm{~m}$ du sol et plus de 3500 pluviomètres (Seguí, 2008 ; Soubeyroux et al. 2008). La chaîne SIM est aussi utilisée pour produire une réanalyse à partir des observations et des réanalyses atmosphériques du CEPMMT (Centre Européen pour les Prévisions Météorologiques à Moyen Terme). La résolution spatio-temporelle est de $8 \mathrm{~km}$ pour l'ensemble du territoire Français. Vidal et al. (2010) ont ainsi pu caractériser les épisodes de sécheresses sur le territoire français comme celui de 2003. 
Les variables utilisées sont d'une part l'indicateur d'humidité du sol, le Soil Wetness Index (SWI mensuel moyen) et, d'autre part, l'évapotranspiration potentielle (ETP), la température à 2 mètres (en moyennes mensuelles) et les précipitations (en cumuls mensuels). Le SWI permet de caractériser la sécheresse des sols. Sur une profondeur d'environ deux mètres, il représente l'état de la réserve en eau du sol par rapport à la réserve utile (eau disponible pour l'alimentation des plantes, calculée à partir de la base de données pédologique au millionième de l'INRAE). Il est généralement noté entre 0 et 1 : lorsque le SWI est égal à zéro, le sol est considéré comme très sec et les végétaux ne peuvent plus en tirer d'eau. Un SWI égal à 1 indique que le sol a atteint sa capacité au champ (Soubeyroux et al., 2011).

Quant à l'ETP, elle permet de suivre l'évolution de la demande évaporatoire de l'atmosphère dont l'augmentation favorise l'asséchement des sols. Elle est fondée sur la formule de Penman-Monteith, méthode de calcul de l'évapotranspiration recommandée par la FAO (Food and Agriculture Organization of the United Nations). Cette formule est aussi utilisée par Météo France pour le calcul de 1'ETP quotidienne (Vannier et Braud, 2012).

La méthodologie employée consiste à étudier et comparer les conditions moyennes et les configurations spatiales des températures, des précipitations, de l'humidité des sols (SWI) et de l'évapotranspiration potentielle (ETP) entre la période 1959-1987 (nommée P1 par la suite) et la période 1988-2019 (P2), pour les différentes saisons. La significativité des différences est testée par un test de Student $(\mathrm{p}=0,05)$. La représentation graphique en «boîte à moustaches» (ou «boxplot») est utilisée afin de décrire et comparer les distributions statistiques des différentes variables étudiées. La ligne centrale de la boîte représente la médiane. Les bords de la boîte correspondent au premier quartile (bord inférieur) et au troisième quartile (bord supérieur). Un quart des valeurs sont inférieures au premier quartile (trois quarts supérieures). A l'inverse, trois quarts des valeurs sont inférieures au troisième quartile (un quart supérieures). Les extrémités des moustaches représentent 1,5 fois l'espace interquartile, soit la distance entre le premier et le troisième quartile. Les valeurs isolées qui se situent en dehors de la boîte à moustaches et qui sont représentées par des croix rouges sont considérées comme exceptionnelles, extrêmes.

\section{Résultats}

\subsection{Variabilité spatio-temporelle des températures et des précipitations en France depuis la rupture climatique de 1987}

La figure 1 montre la différence des températures entre les périodes $\mathrm{P} 1$ et $\mathrm{P} 2$ pour les quatre trimestres de l'année. La hausse des températures est généralisée. Les surfaces grisées indiquent que les différences sont significatives au seuil $p=0,05$ selon le test de Student sur l'ensemble du territoire.

Les différences les plus élevées se rencontrent au printemps avec un maximum de $3^{\circ} \mathrm{C}$, ainsi qu'en été pour toute la partie sud et est du territoire qui montrent les plus fortes augmentations (entre 1 et $3^{\circ} \mathrm{C}$ ). La vallée du Rhône semble être la région française où les températures augmentent le plus entre P1 et P2 (figure 1). Les boîtes à moustaches de la figure 2 permettent de comparer les distributions saisonnières des températures entre les périodes P1 et P2. L'hiver et l'automne présentent les plus faibles augmentations de températures entre les deux périodes, la médiane passant de $3,3^{\circ} \mathrm{C}$ en hiver $\mathrm{P} 1$ à $4,4^{\circ} \mathrm{C}$ en hiver $\mathrm{P} 2$ (soit un réchauffement de $1,1^{\circ} \mathrm{C}$ ). En automne, l'augmentation est du même ordre de grandeur puisque la médiane passe de $9,8^{\circ} \mathrm{C}$ en $\mathrm{P} 1$ à $10,8^{\circ} \mathrm{C}$ en $\mathrm{P} 2$, soit une hausse de $1^{\circ} \mathrm{C}$. Lors de ces deux saisons, il semblerait que, tout comme en été et au printemps, la France soit partagée selon une diagonale sud-ouest / nord-est avec une partie est ayant subi les plus fortes augmentations (notamment le sud-ouest et le nord-est ; figure 1). A l'inverse, des régions de l'ouest, comme la Bretagne, présentent des réchauffements moins importants, généralement compris entre 0,5 et $1^{\circ} \mathrm{C}$ selon les saisons (figure 1). Il est important de souligner que les décalages des valeurs médianes vers les températures plus chaudes sont associés à des dispersions dont les principales caractéristiques restent quasi similaires entre $\mathrm{P} 1$ et $\mathrm{P} 2$, et notamment des espaces interquartiles quasi inchangés (figure 2). 

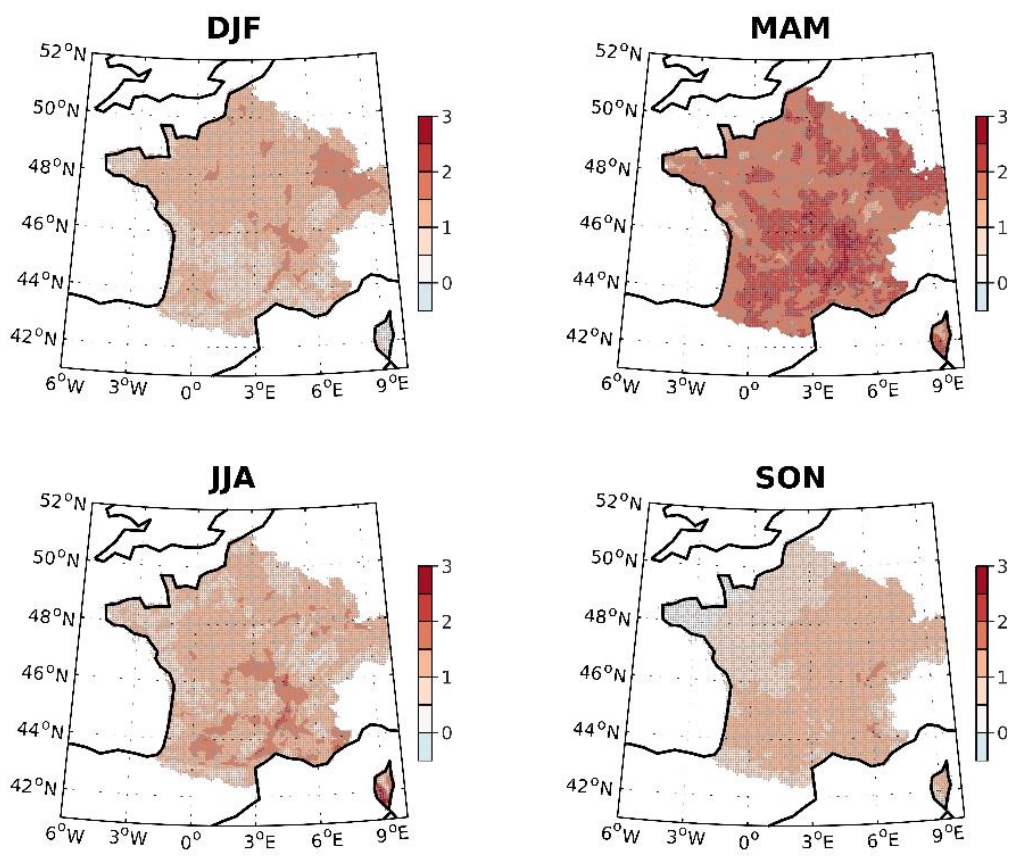

Figure 1. Différences de températures saisonnières moyennes pour chaque point de la grille SAFRAN entre deux souspériodes: P2 (1988-2019) - P1 (1959-1987). Les différences sont exprimées en degrés Celsius. Les zones pour lesquelles les différences sont statistiquement significatives par un test de Student $(p=0,05)$ sont indiquées en grisé. Differences is mean seasonal temperatures for each SAFRAN grid points between two sub-periods: P2 (1988-2019) $P 1$ (1959-1987). Differences are in Celsius degrees. The significant differences (Student's $T$-Test, $p=0.05)$ change is indicated in shaded gray.

Figure 2. Boxplots des températures saisonnières moyennes en France (réalisés à partir de tous les points de la grille SAFRAN) pour deux sous-périodes: P1 (1959-1987) et P2 (1988-2019) en hiver (DJF), au printemps (MAM), en été (JJA) et en automne (SON). Les températures sont exprimées en degrés Celsius. Boxplots of seasonal mean temperatures of France (from all SAFRAN grid points) for two sub-periods: P1 (19571987) and $P 2$ (1988-2019) in winter (DJF), spring (MAM), summer (JJA) and autumn (SON). Temperatures are in Celsius degrees.

Le réchauffement climatique semble donc principalement s'exprimer par un glissement généralisé des distributions vers des températures plus élevées, et donc un climat moyen de plus en plus chaud. Ces résultats complètent de nombreuses autres études réalisées sur les séries de températures en France montrant une augmentation des températures pour toutes les saisons depuis les années 1950 (Moisselin et al., 2002 ; Gibelin et al.,

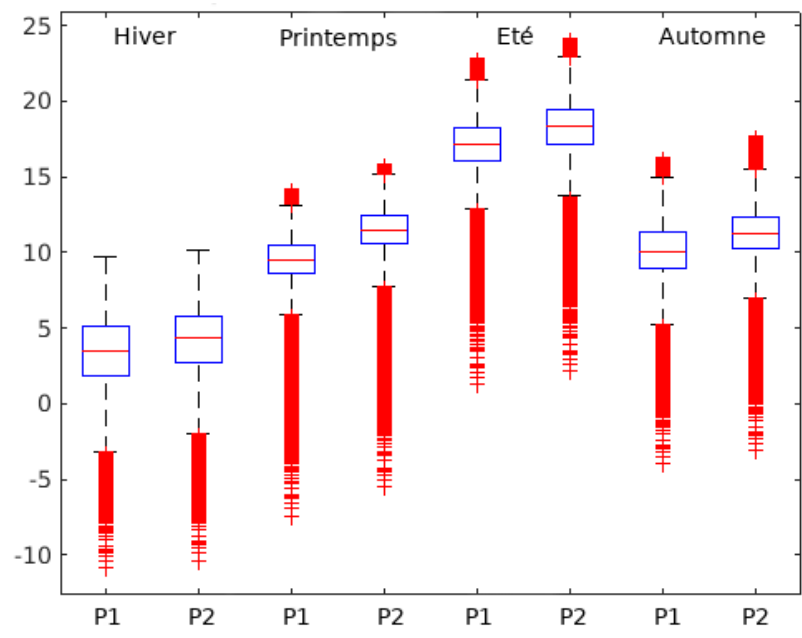

2014 ; Brulebois et al., 2015), particulièrement au printemps et en été (Gibelin et al., 2014).

En ce qui concerne les précipitations, la figure 3 présentent la différence de cumuls de précipitation entre $\mathrm{P} 1$ et $\mathrm{P} 2$ pour chacun des quatre trimestres de l'année. A l'échelle de l'ensemble de la France, les cumuls saisonniers de pluie sont quasiment similaires entre les deux sous-périodes. Seul l'automne (SON) semble connaître une hausse du 
cumul de pluie, passant de $80 \mathrm{~mm}$ en moyenne sur la période 1959-1987 à $87 \mathrm{~mm}$ sur la période 19882019 (figure 3). Si cette hausse n'est pas statistiquement significative à l'échelle du pays, elle le devient au cours de l'automne et de l'hiver au sud-est et sur l'arc Alpin (non montré). Il en va de même pour les valeurs médianes et les percentiles $(25,50$ et 75$)$ qui ne montrent aucun changement significatif entre $\mathrm{P} 1$ et $\mathrm{P} 2$. Ces résultats corroborent ceux de Brulebois et al. (2015) indiquant qu'aucune

Figure 3. Boxplots des cumuls saisonniers moyens de précipitations en France (réalisés à partir de tous les points de la grille SAFRAN) pour deux sous-périodes : P1 (1959-1987) et P2 (1988-2019) en hiver (DJF), au printemps (MAM), en été (JJA) et en automne (SON). Les cumuls sont exprimés en $\mathrm{mm}$. Boxplots of seasonal mean rainfall amount of France (from all SAFRAN grid points) for two sub-periods: $P 1$ (1957-1987) and P2 (1988-2019) in winter (DJF), spring (MAM), summer (JJA) and autumn (SON). Rainfall amounts are in Celsius $\mathrm{mm}$.

\subsection{Evolution spatiale et saisonnière de l'humidité des sols en France}

L'humidité des sols (SWI) a elle aussi évolué après 1987 (figures 4 et 5) et affiche une forte diminution du printemps à l'automne. L'assèchement est particulièrement marqué dans le sud et le sudouest, principalement au printemps et en été (figure 4). Au printemps, l'aggravation des sécheresses des sols est la plus prononcée dans le quart-sud-ouest de la France alors qu'en été, c'est le quart nord-est qui est le plus touché (figure 4). A la différence des températures qui montrent une hausse significative sur l'ensemble du territoire, la variabilité spatiale du SWI est un peu plus contrastée et univoque. Même si les différences P1-P2 sont majoritairement négatives à l'échelle de la France (témoignant d'assèchement des sols), elles peuvent parfois être positives comme c'est le cas en hiver sur les reliefs de l'est mais aussi sur certains secteurs au nord de la France, la Bretagne ou la façade est (figure 4). Il faut cependant noter que ces évolutions positives ne rupture de stationnarité n'a été détectée dans les précipitations moyennes en France depuis 1969. Cela ne signifie pas obligatoirement que le changement climatique n'ait pas affecté les précipitations, ce qui pourrait notamment être le cas aux échelles intra-saisonnières ou interannuelles, mais que la brusque hausse de température observée en 1987-1988 en France ne s'est pas accompagnée d'un changement significatif des conditions saisonnières moyennes des précipitations.

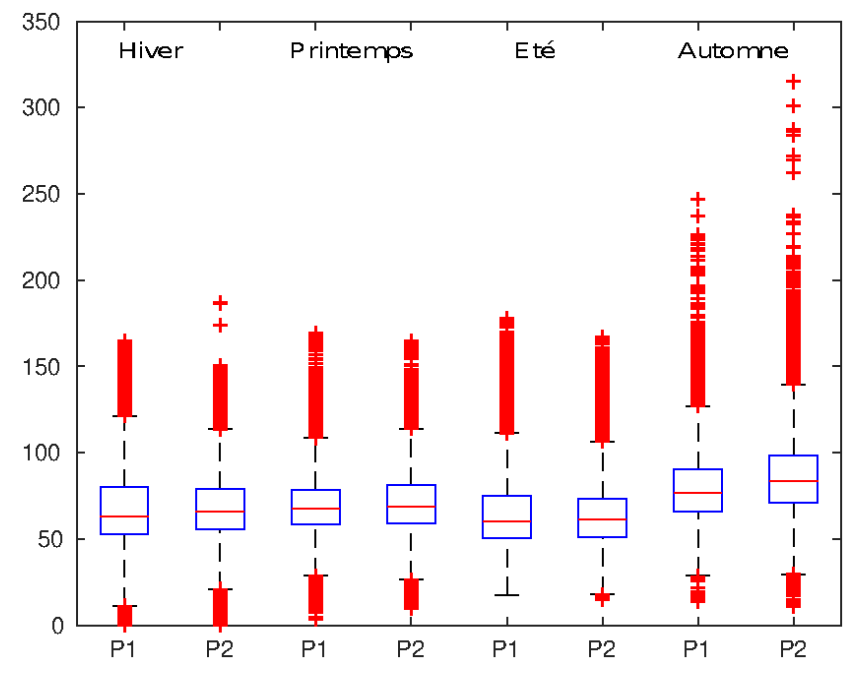

sont pas toujours statistiquement significatives hormis dans le massif alpin en hiver. Sur ces régions, l'augmentation de l'humidité des sols en hiver et moindrement au printemps se produit en raison d'une augmentation des précipitations sur ces reliefs (non montré).

A l'échelle de la France, l'évolution du SWI entre les deux périodes étudiées diffère dans ses caractéristiques en fonction des saisons (figure 5). En hiver, la valeur médiane ne change pratiquement pas, passant de 0,88 en $\mathrm{P} 1$ à 0,87 en $\mathrm{P} 2$. En revanche, l'étendue des valeurs au-delà et en deçà de 1,5 fois l'espace interquartile est plus élevée (figure 4). Autrement dit, les caractéristiques moyennes d'humidité du sol restent les mêmes en $\mathrm{P} 2$ par rapport à $\mathrm{P} 1$ mais des situations de plus en plus « extrêmes » peuvent se produire en terme de déficit ou de surplus d'humidité des sols. Ces changements aussi bien dans les fortes et faibles valeurs du SWI expliquent, qu'en hiver, le sudouest de la France connaît des situations de 
sécheresse de plus en plus aggravées alors que les sols $\mathrm{du}$ centre-est et $\mathrm{du}$ nord-est sont significativement plus humide en P2 qu'en P1 (figures 4 et 5). Au printemps et en été, le SWI médian baisse significativement entre P1 et P2 (ainsi que la valeur moyenne, non montrée) et la gamme des valeurs de SWI semble aller vers des sols de plus en plus secs (figure 5). Ces
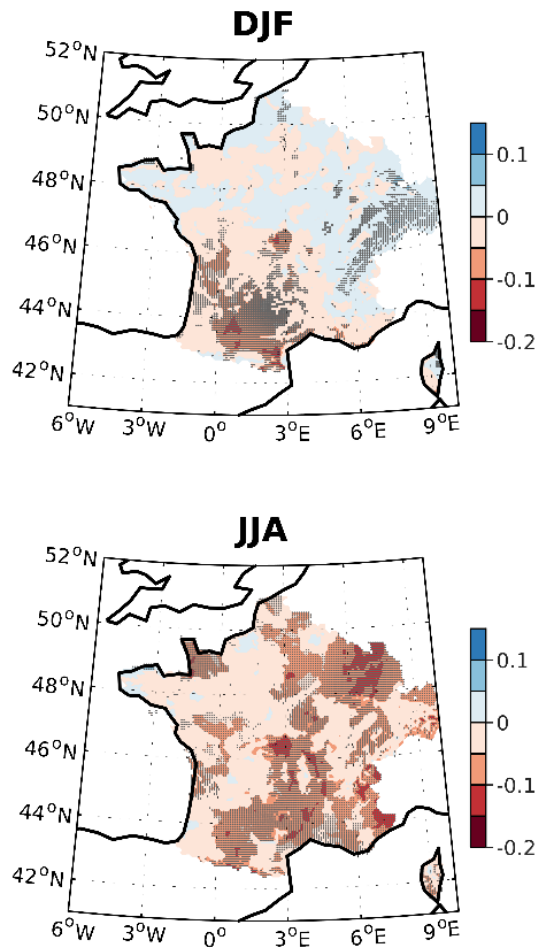

changements indiquent que les printemps et les étés ont subi un assèchement moyen généralisé sur la quasi-totalité du territoire avec des aggravations des situations des sécheresses fortes à sévères, notamment dans le nord-est et le sud-ouest de la France ainsi que le pourtour méditerranéen (figures 4 et 5$)$.
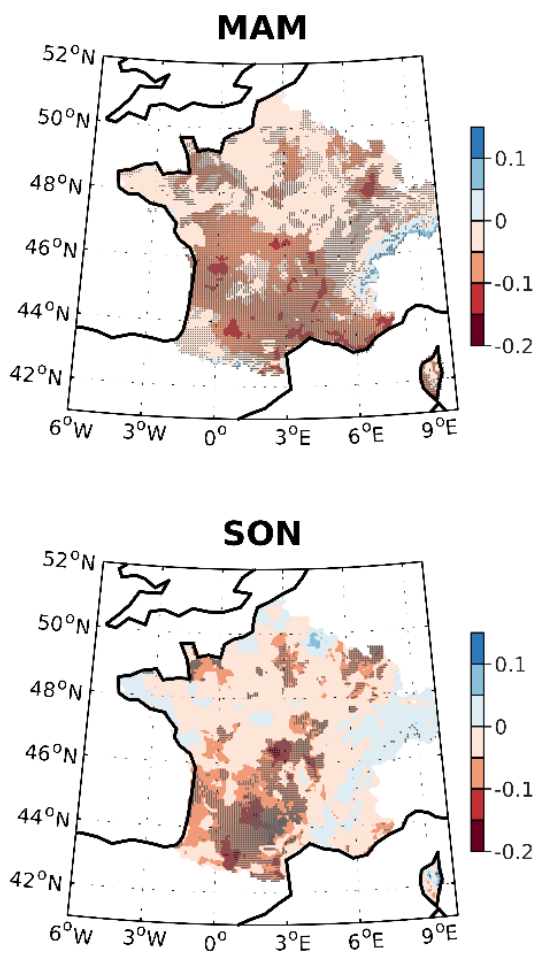

Figure 4. Différences de SWI saisonniers moyens pour chaque point de la grille SAFRAN entre deux sous-périodes : P2 (1988-2019) - P1 (1959-1987). Les zones pour lesquelles les différences sont statistiquement significatives par un test de Student $(p=0,05)$ sont indiquées en grisé. Differences is mean seasonal SWI for each SAFRAN grid points between two sub-periods: P2 (1988-2019) - P1 (1959-1987). The significant differences (Student's T-Test, $p=0.05)$ change is indicated in shaded gray.

Figure 5. Boxplots des SWI saisonniers moyens en France (réalisés à partir de tous les points de la grille SAFRAN) pour deux sous-périodes : P1 (1959-1987) et P2 (1988-2019) en hiver (DJF), au printemps (MAM), en été (JJA) et en automne (SON). Boxplots of seasonal mean SWI of France (from all SAFRAN grid points) for two sub-periods: P1 (1957-1987) and P2 (1988-2019) in winter (DJF), spring (MAM), summer (JJA) and autumn (SON).

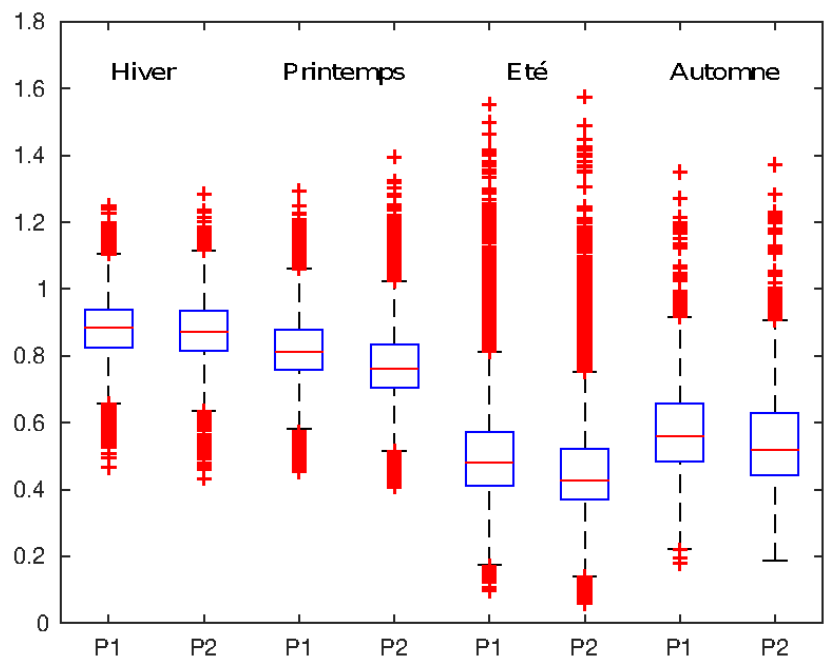


Pour résumer, l'évolution du SWI n'est pas identique sur tout le territoire en raison des conditions climatiques qui évoluent différemment aux échelles régionales. Néanmoins, pour l'ensemble des saisons, les températures moyennes augmentent après 1987, pouvant expliquer pourquoi les sols s'assèchent. Les cumuls saisonniers moyens des précipitations ne montrant pas de différences significatives entre P1 et $\mathrm{P} 2$, la diminution du SWI moyen après le saut des températures de 1987 pourrait donc être principalement expliquée par la hausse induite de la demande évaporatoire de l'atmosphère, couplée à une diminution des précipitations lors de certains mois. En effet, certains auteurs ont montré que les cumuls mensuels de pluie en été tendent à diminuer en France au cours du $20^{\mathrm{e}}$ siècle (Moisselin et al., 2002).

\subsection{Demande évaporatoire de l'atmosphère}

L'évapotranspiration potentielle est un indicateur important pour l'agronomie puisque sa

Figure 6 : Boxplots des ETP saisonniers moyens en France (réalisés à partir de tous les points de la grille SAFRAN) pour deux sous-périodes : P1 (1959-1987) et P2 (1988-2019) en hiver (DJF), au printemps (MAM), en été (JJA) et en automne (SON). Boxplots of seasonal mean PET of France (from all SAFRAN grid points) for two sub-periods: P1 (1957-1987) and P2 (1988-2019) in winter (DJF), spring (MAM), summer (JJA) and autumn $(S O N)$.

Cette augmentation de l'ETP est extrêmement forte au printemps et en été et dans une moindre mesure, en hiver (figures 6 et 7). Au printemps, la valeur médiane de l'ETP passe de $2,11 \mathrm{~mm} / \mathrm{j}$ pour $\mathrm{P} 1$ à 2,63 mm/j pour P2 (figure 6). En été, elle passe de 3,51 pour $\mathrm{P} 1$ à 3,87 pour $\mathrm{P} 2$. En outre, pour ces deux saisons, les fortes et très fortes valeurs de l'ETP tendent à devenir de plus en plus élevées, témoignant d'une exacerbation des évènements de très fortes demandes en eau de l'atmosphère. La période la plus récente (P2) a donc subi un changement drastique d'état moyen de la demande valeur correspond au volume d'eau nécessaire pour une croissance optimale de la végétation. Cette variable dépend de plusieurs paramètres, principalement de la température et du rayonnement solaire mais aussi de la vitesse du vent ainsi que l'humidité de l'air.

L'une des principales questions est de savoir comment évolue la demande évaporatoire de l'atmosphère sous l'effet du changement climatique contemporain et notamment de la hausse des températures décrite dans la section 2.1 , et quels sont les liens potentiels avec les caractéristiques spatio-temporelles de l'assèchement des sols présentées dans la section 2.2. Les résultats de la figure 6 montrent que l'évapotranspiration potentielle a augmenté entre $\mathrm{P} 1$ et $\mathrm{P} 2$ pour l'ensemble des saisons à l'exception de l'automne (qui montre en moyenne une baisse de l'ETP ; figures 6 et 7).

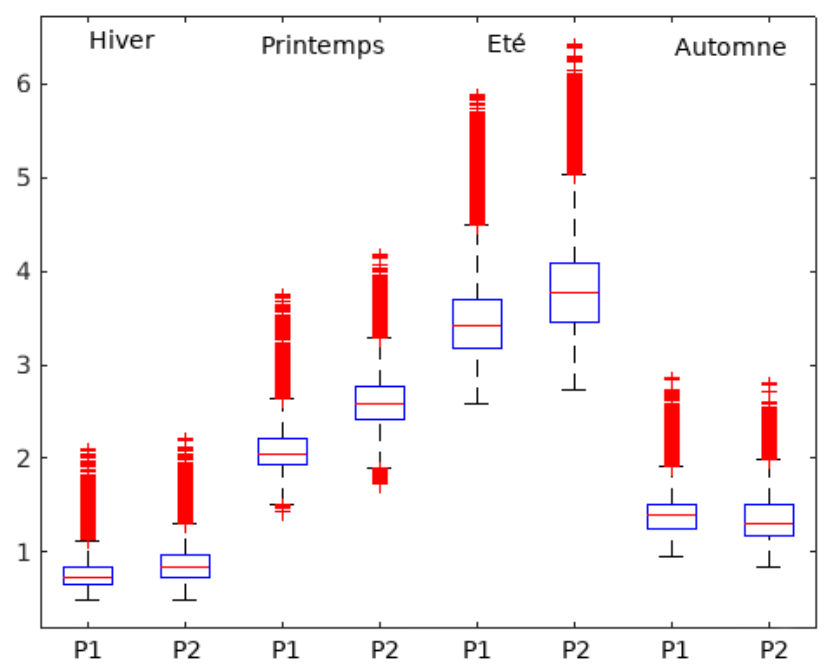

évaporatoire de l'atmosphère ainsi qu'une aggravation des conditions extrêmes au printemps et en été. Ces changements sont en phase temporellement et spatialement avec les importantes hausses des températures au printemps et en été (figures 1 et 7). Ces dernières démontrent également qu'un réchauffement des températures au-delà d'un certain seuil peut entraîner une réponse non linéaire en termes d'évapotranspiration potentielle, et même une réponse exacerbée qui pourrait presque ici ressembler à un mécanisme « d'emballement» au printemps et en été. 
Bien que les différences soient statistiquement significatives sur toute la France et pour toutes les saisons, certaines parties du territoire semblent subir des changements plus importants que d'autres. En effet, même si au printemps et en été toute la
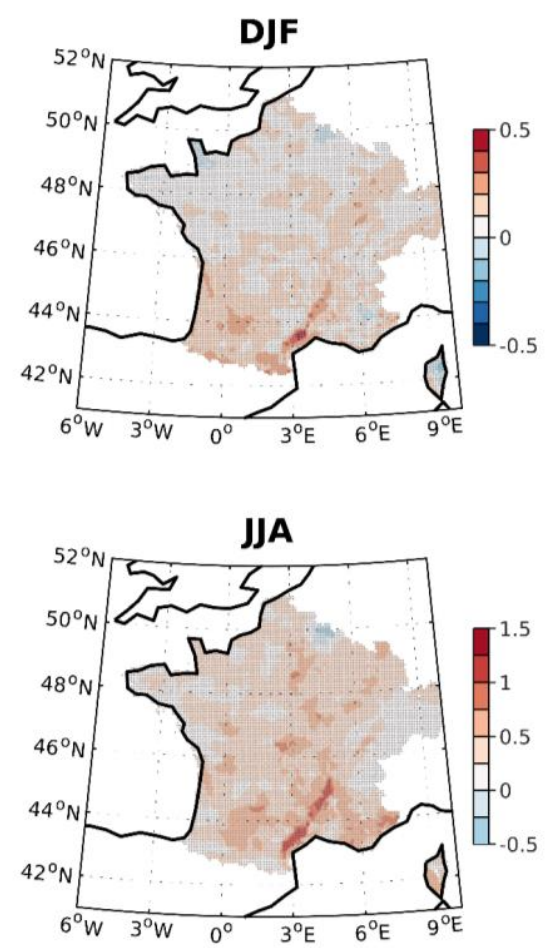

France voit 1'ETP augmenter entre 0,5 et $1 \mathrm{~mm} / \mathrm{j}$ entre les deux périodes, c'est surtout la partie sud, notamment le sud-ouest, le pourtour méditerranéen ainsi que dans la vallée du Rhône, qui connaît les plus fortes augmentations de l'ETP (figure 7).
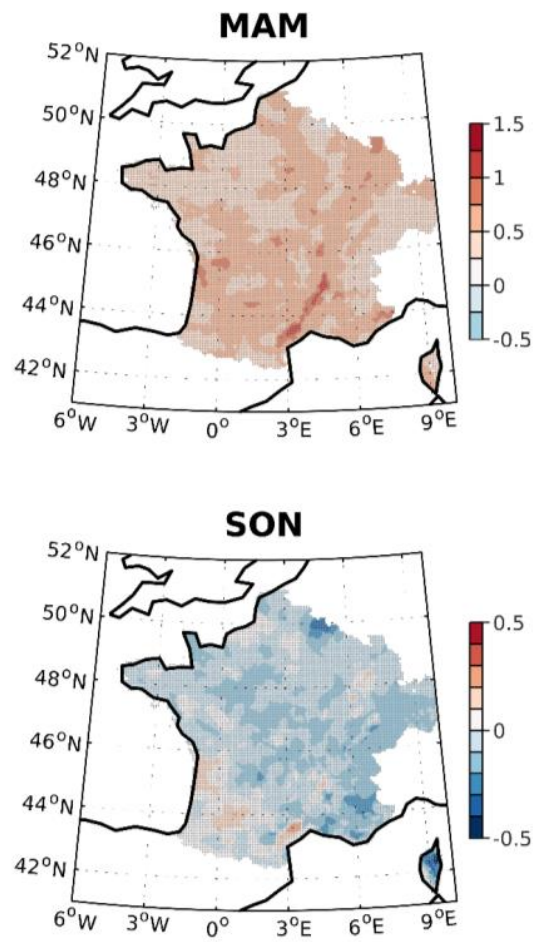

Figure 7. Différence d'ETP saisonniers moyens pour chaque point de la grille SAFRAN entre deux sous-périodes : P2 (1988-2019) - P1 (1959-1987). Les zones pour lesquelles les différences sont statistiquement significatives par un test de Student $(p=0,05)$ sont indiquées en grisé. Differences is mean seasonal PET for each SAFRAN grid points between two sub-periods: P2 (1988-2019) - P1 (1959-1987). The significant differences (Student's T-Test, $p=0.05)$ change is indicated in shaded gray.

L'explication vient sans doute de l'importante élévation des températures entre P1 et P2 au printemps et en été pour ces différents espaces méridionaux. Par exemple, la vallée du Rhône illustre bien le lien important entre l'augmentation des températures et de l'évapotranspiration potentielle en cumulant la plus forte augmentation des températures du territoire français $\left(+3^{\circ} \mathrm{C}\right)$ et la plus forte hausse de la demande évaporatoire de l'atmosphère $(+1,5 \mathrm{~mm})$ (figures 1 et 7$)$. Aux échelles plus fines, des secteurs localisés et bien délimités montrent des augmentations de l'ETP particulièrement importantes. C'est notamment le cas de la plaine de Woëvre qui est le secteur le moins arrosé et le plus chaud de l'espace regroupant la Meuse, la Moselle et la Meurthe-et-Moselle, en raison des caractéristiques topographiques singulières et de l'effet d'abris conséquent. C'est également le cas de l'arc Cévenol allant de l'Aude à la vallée du Rhône. Ce secteur qui cumule des conditions d'ensoleillement et de vent parmi les plus importantes de France a également connu un réchauffement printanier et estival parmi les plus élevé du territoire national depuis les années 1950 (figures 1 et 7). Les caractéristiques géographiques locales semblent donc être des facteurs particulièrement aggravants de l'effet du réchauffement sur la demande évaporatoire. Les résultats montrent que l'augmentation de la demande évaporatoire de l'atmosphère peut être directement liée à la hausse des températures, tandis que les cumuls précipités n'ont pas statistiquement 
évolués. Pour la végétation, cela peut se traduire par une augmentation des besoins en eau et des épisodes de sécheresses végétales/agricoles menaçant les rendements. Si l'augmentation des températures est un fait établi, l'évolution des autres variables atmosphériques impliquées dans l'évapotranspiration est moins évidente à déterminer. Le rayonnement solaire ainsi que la vitesse des vents sont des variables à prendre en compte dans le calcul de l'évapotranspiration potentielle. Moisselin et Canellas (2005) ont montré une tendance à la diminution de l'ensoleillement en France entre 1931 et 2000 sur la quasi-totalité du territoire hormis certains secteurs du pourtour méditerranéen. Concernant les vents, les travaux de McVicar et al. (2012) à l'échelle de l'Europe et ceux de Jourdier (2015) pour la France présentent des tendances à la diminution de la vitesse annuelle moyenne des vents entre 1950 et 2000 . Ces résultats confortent la prépondérance de la hausse des températures dans la l'augmentation de la demande évaporatoire de l'atmosphère et de l'assèchement des sols.

\section{Discussion et conclusion}

Le réchauffement significatif des températures en France entre les deux sous-périodes étudiées et la hausse de la demande évaporatoire de l'atmosphère semblent être deux facteurs couplés pouvant expliquer l'assèchement des sols, notamment au printemps et en été. Nos résultats ont montré que l'augmentation rapide des températures en France après 1987 a contribué à très fortement augmenter la demande évaporatoire de l'atmosphère avec notamment une importante aggravation des très fortes demandes évaporatoires, surtout au printemps et en été. A l'échelle du territoire français, les précipitations semblent rester stationnaires sur toute la période 1959-2019 au vu de l'analyse de l'évolution des cumuls saisonniers moyens à l'exception des reliefs alpins. Toutefois, cette stationnarité n'exclue pas des changements dans les caractéristiques intra-saisonnières telles que des changements dans les cycles saisonniers moyens (Moisselin et al., 2002) ou dans les répartitions entre les jours secs et jours pluvieux, notamment les longues séquences sèches (Raymond et al., 2019). Ces changements drastiques des conditions d'évapotranspiration potentielle associés au réchauffement des températures amènent des sols plus secs au printemps et en été, aggravant ainsi le risque de sécheresses végétales et agricoles. En effet, une conséquence directe de l'augmentation des températures est l'accélération du cycle hydrologique. Un fort réchauffement comme celui observé au printemps et en été induit une augmentation très importante de l'énergie et du contenu en eau de l'atmosphère, qui a pour conséquence une accélération des flux d'humidité et notamment de l'évapotranspiration (PascoliniCampbell et al., 2021). Les mêmes auteurs soulignent que la demande évaporatoire de l'atmosphère étant de plus en plus forte, une part croissante des précipitations alimente le processus d'évaporation, diminuant de fait les écoulements, asséchant ainsi les sols au printemps et en été, aggravant le risque de sécheresses agricoles et hydrologiques.

Spatialement, c'est surtout le nord-est de la France, le sud-ouest et le pourtour Méditerranéen qui sont les plus fortement touchés par le réchauffement des températures et ses effets induits sur l'évapotranspiration et l'humidité des sols. En outre, aux échelles plus fines, les caractéristiques géographiques locales, surtout l'orographie, peuvent être des facteurs aggravant de ces effets induits du réchauffement sur l'évapotranspiration, notamment via l'orientation, les effets d'abris et la canalisation des vents. Certains secteurs sont donc des «hotspots» de l'effet du réchauffement, comme par exemple la plaine de la Woëvre en Moselle ou les contreforts des Cévennes et les Alpes Maritimes.

Depuis les années 2000, douze années sur dixsept ont été touchées par des sécheresses aggravées du sol. Au $21^{\mathrm{e}}$ siècle, les simulations indiquent une hausse importante de la fréquence des épisodes de sécheresses du sol, surtout à partir de 2050 (Soubeyroux et al., 2012). Nos travaux de recherches ont permis de montrer que le réchauffement généralisé des températures s'exprime certes par un assèchement moyen des sols mais également par des épisodes de sols très secs à extrêmement secs de plus en plus marqués, et que le printemps et l'été sont des saisons 
particulièrement touchées. Ces travaux ont par ailleurs mis en lumière des secteurs déjà particulièrement sensibles face à l'assèchement des sols depuis les années 1950, comme le sud-ouest et le nord-est de la France.

Depuis les années 1990, la France connaît une stagnation des rendements des principales cultures en raison notamment des modifications du cycle de l'eau et de l'assèchement des sols (Ben-Ari et al., 2018 ; Schauberger et al., 2018) L'aggravation du risque climatique sur l'agriculture, et surtout celui associé aux sécheresses, est particulièrement surveillé en raison de la multiplication de cet aléa et de l'aggravation des conséquences sur l'agriculture. En effet, depuis 2021, le gouvernement français a réformé l'assurance récolte pour faire face aux conséquences du changement climatique et mieux protéger les exploitants tant la vulnérabilité du monde agricole semble s'accentuer. Outre ces mesures de protection des exploitants au travers les assurances, le changement climatique va nécessiter des mesures d'adaptation territorialisées au regard des changements plus marqués pour certaines parties du pays, de leurs caractéristiques géographiques, et des activités agricoles les plus sensibles.

Remerciements: Les résultats présentés dans cet article sont issus du travail de recherche de Lisa Sick effectué dans le cadre de son stage de master 2 SEME (Sol, Eau, Milieux, Environnement). Il a été financé par l'équipe CRC (Centre de Recherche de Climatologie) de l'UMR Biogéosciences de l'Université de Bourgogne que nous tenons à remercier.

\section{Références}

Amigues J.-P., Debaeke P., Itier B., Lemaire G., Seguin B., Tardieu F., Thomas A., 2006. Sécheresse et agriculture. Réduire la vulnérabilité de l'agriculture à un risque accru de manque d'eau. Expertise scientifique collective. Rapport, INRA, 380 p. + annexes. ffhal-03167191

Ben-Ari T., Boé J., Ciais P., Lecerf R., Van der Velde M., Makowski D., 2018. Causes and implications of the unforeseen 2016 extreme yield loss in the breadbasket of France. Nat. Communication, 9, 1627. doi.org/10.1038/s41467018-04087-x

Brulebois E., Castel T., Richard Y., Chateau-Smith C., Amiotte-Suchet P., 2015. Hydrological response to an abrupt shift in surface air temperature over France in 1987/1988. Journal of Hydrology, 531, 892-901. 10.1016/j.jhydrol.2015.10.026.

Cassou C., 2004. Du changement climatique aux régimes de temps : l'oscillation nord-atlantique. Météorologie, 45, 21-32. $10.4267 / 2042 / 36039$

Forzieri G., Feyen L., Russo S., Vousdoukas M., Alfieri L., Outten S., Migliavacca M., Bianchi, A., Rojas R., Cid A., 2016. Multi-hazard assessment in Europe under climate change. Climatic Change, 137, 105-119. 10.1007/s10584016-1661-X.

Gibelin A.-L., 2015. Évolution du climat passé en France métropolitaine depuis les années 1950. [en ligne], http://www.meteo.fr/cic/meetings/2015/agrometeo/document s/1_01.pdf.

Habets F., Boone A., Champeaux J.-L., Etchevers P., Franchistéguy L., Leblois E., Ledoux E., Moigne P.-L., Martin E., Morel S., Noilhan J., Seguí P. Q., Rousset-Regimbeau F., Viennot P., 2008. The SAFRAN-ISBA-MODCOU hydrometeorological model applied over France. Journal of Geophysical Research: Atmospheres, 113. 10.1029/2007JD008548.

IPCC, 2013. Climate Change 2013. The Physical Science Basis. Cambridge Universirty Press, Cambridge, United Kingdom and New-York, NY, USA, 1535 p.

IPCC, 2021. Climate Change 2021: The Physical Science Basis. Contribution of Working Group I to the Sixth Assessment Report of the Intergovernmental Panel on Climate Change.Cambridge University Press.

Jourdier B., 2015. Ressource éolienne en France métropolitaine : méthodes d'évaluation du potentiel, variabilité et tendance. Thèse de doctorat de l'école Polytechnique laboratoire de météorologie dynamique, 187 p.

Mc Vicar T. R., Roderick M. L., Donohue R. J., Li L. T., Van Niel T. G., Thomas A., Grieser J., Jhajharia D., Himri Y., Mahowald N. M., Mescherskaya A. V., Kruger A. C., Rehman S., Dinpashoh Y., 2012. Global review and synthesis of trends in observed terrestrial near-surface wind speeds: Implications for evaporation. Journal of Hydrology, 416-417, 182-205. 10.1016/j.jhydrol.2011.10.024.

Moisselin J.-M., Schneider M., Canellas C., Mestre O., 2002. Les changements climatiques en France au $\mathrm{XX}^{\mathrm{e}}$ siècle : étude des longues séries homogénéisées de données de température et de précipitations. La Météorologie, 38, 45-56.

Moisselin J.-M., Canellas C., 2005. French homogenized sunshine-duration long-term series. C. R. Géoscience, 3337, 8, 729-734. 10.1016/j.crte.2005.04.002.

Pascolini-Campbell M., Reager J. T., Chandanpurkar H. A., Rodell M., 2021. A 10 per cent increase in global land evapotranspiration from 2003 to 2019. Nature, 593, 543547. doi.org/10.1038/s41586-021-03503-5.

Rapport AGRESTE, 2018. Une sécheresse qui s'accentue en septembre. Agreste Infos Rapides-Climatologie, octobre 2018, 147 p. 
Rapport AGRESTE, 2019. Sécheresse et record de chaleur en juillet. Agreste Infos Rapides - Climatologie, août 2019, 115 p.

Rapport AGRESTE, 2020. Sécheresse marquée en juillet. Agreste Infos Rapides - Climatologie, août 2020, 108 p.

Raymond F., Ullmann A., Camberlin P., 2018. Très longs épisodes secs hivernaux dans lebassin méditerranéen : variabilité spatio-temporelle et impact sur la production céréalière en Espagne, Cybergeo: European Journal of Geography, document 858. doi.org/10.4000/cybergeo.29156.

Raymond F., Ullmann A., Tramblay Y., Drobinsky P., Camberlin P., 2019. Evolution of Mediterranean extreme dry spells 2 during the wet season under climate change. Regional Environmental Change, 19, 2339-2351. doi.org/10.1007/s10113-019-01526-3.

Seguí P. Q., 2008. Simulation hydrologique en région méditerranéenne avec SAFRAN-ISBA-MODCOU. Amélioration de la physique et évaluation des risques dans le cadre du changement climatique. Hydrologie. Université Paul Sabatier - Toulouse III, 〈tel-00367576〉

Schauberger B., Ben-Ari T., Makowski D., Tomomichi K., Ciais P., 2018. Yield trends, variability and stagnation analysis of major crops in France over more than a century. Sci. Rep., 8, 16865. doi.org/10.1038/s41598-018-35351-1.

Seo J., Shim S., Kwon S.-H. and coauthors, 2020. The impacts of aerosol emissions on historical climate in UKESM1. Atmosphere, 11, 1095. doi.org/10.3390/atmos11101095.

Soubeyroux J.-M., Martin E., Franchistéguy L., Habets F., Noilhan J., Baillon M., Regimbeau F., Vidal J.-P., Le Moigne P., Morel S., 2008. SAFRAN-ISBA-MODCOU (SIM) - Un outil pour le suivi hydrométéorologique opérationnel et les études. La Météorologie, 8, 63, 40-45.

Soubeyroux J.-M, Vidal J.P., Najac J., Kitova N., Blanchard M., Dandin P., Martin E., Pagé C., Habets F., 2011. Projet ClimSec. Impact du changement climatique en France sur la sécheresse et l'eau du sol. Rapport final. Irstea, 72 P. 〈hal02595492>

Soubeyroux J.-M., Kitova N., Blanchard M., Vidal J.-P., Martin É. and Dandin P., 2012. Sécheresses des sols en France et changement climatique : Résultats et applications du projet ClimSec. La Météorologie, 8-21. 10.4267/2042/47512.

Van der Velde M., Tubiello F. N., Vrieling A., Bouraoui, 2012. Impacts of extreme weather on wheat and maize in France: evaluating regional crop simulations against observed data. Climatic Change, 113, 751-765. doi.org/10.1007/s10584011-0368-2.

Vannier O., Braud I., 2012. Calcul d'une évapotranspiration de référence spatialisée pour la modélisation hydrologique à partir des données de la réanalyse SAFRAN de Météo-France. [Rapport de recherche] Irstea, 22 p. 〈hal-02593413〉

Vidal J.-P., Martin E., Franchistéguy L., Habets F., Soubeyroux J.-M., Blanchard M., Baillon M., 2010. Multilevel and multiscale drought reanalysis over France with the Safran-IsbaModcou hydrometeorological suite. Hydrology and Earth System Sciences, 14, 459-478. 10.5194/hess-14-459-2010.

Vidal J.-P., Martin E., Kitova N., Najac J., Soubeyroux J.-M., 2012. Evolution of spatio-temporal drought characteristics: validation, projections and effect of adaptation scenarios. Hydrology and Earth System Sciences, 16, 8, 2935-2955.

Citation de l'article : Sick L. et al., 2021. La rupture climatique de 1987 en France : quels effets sur l'humidité des sols ? Climatologie, $18,5$. 